\title{
Impact of Band Nonparabolicity on Threshold Voltage of Nanoscale SOI MOSFET
}

\author{
Yasuhisa Omura \\ ORDIST, Grad. School of Sci. \& Eng., Kansai University, 3-3-35 Yamate-cho, Suita, \\ Osaka 564-8680, Japan \\ Correspondence should be addressed to Yasuhisa Omura; omuray@kansai-u.ac.jp
}

Received 24 September 2016; Revised 13 November 2016; Accepted 16 November 2016

Academic Editor: Mingxiang Wang

Copyright (C) 2016 Yasuhisa Omura. This is an open access article distributed under the Creative Commons Attribution License, which permits unrestricted use, distribution, and reproduction in any medium, provided the original work is properly cited.

\begin{abstract}
This paper reconsiders the mathematical formulation of the conventional nonparabolic band model and proposes a model of the effective mass of conduction band electrons including the nonparabolicity of the conduction band. It is demonstrated that this model produces realistic results for a sub-10-nm-thick Si layer surrounded by an $\mathrm{SiO}_{2}$ layer. The major part of the discussion is focused on the low-dimensional electron system confined with insulator barriers. To examine the feasibility of our consideration, the model is applied to the threshold voltage of nanoscale SOI FinFETs and compared to prior experimental results. This paper also addresses a model of the effective mass of valence band holes assuming the nonparabolic condition.
\end{abstract}

\section{Introduction}

In the last 3 decades, silicon-on-insulator (SOI) MOSFETs have been attracting attention because of their high shortchannel effect immunity [1] and excellent potential with regard to future nanoscale devices [2]. Therefore, many studies on quantum confinement effects have been performed. The author predicted that the threshold voltage $\left(V_{\mathrm{th}}\right)$ of ultrathin-body (UTB) SOI MOSFET rises as SOI body thickness $\left(t_{S}\right)$ is reduced due to the quantum-mechanical confinement effect [3]. Several studies have demonstrated experiments [4] that, they believe, demonstrate the effect of quantum confinement. However, the author demonstrated that the apparent rise of $V_{\text {th }}$ of UTB SOI MOSFETs is due to more than the quantum effect; it includes the semiclassical effect [5]. Other experiments strongly suggested that a simple parabolic band model is not appropriate in analyzing $V_{\text {th }}$ of nanoscale SOI MOSFETs [6, 7]; in these experiments, the threshold voltage was lower than the simulation values that assume the parabolic conduction band ( $X$ bands for $\mathrm{Si}$ ) for $t_{S}<5 \mathrm{~nm}$. The possibility of determining the limits of the conventional parabolic band approximation is a motivation of this study. Recently, a couple of research articles demonstrated the importance of band nonparabolicity in the analysis of the transport characteristics of nanoscale materials $[8,9]$, where the first principle calculation and the tightbinding method are used to compute the electronic states. In practical situations, however, demand an analytical closed form for the quantized energy levels and an effective mass tensor is requested for electronic device designs, since they greatly reduce the time costs.

This paper reconsiders the mathematical formulation of the conventional nonparabolic band model. This paper examines whether some perturbations can be added to the conventional model for convenience. In the following discussion, this paper focuses on a low-dimensional electron system confined by insulator barriers. We discuss the impact of the nonparabolic conduction band in $\mathrm{Si}$ on the effective mass and propose an analytical expression for the effective mass of electrons including the conduction band nonparabolicity. The model is applied to the threshold voltage of nanoscale SOI FinFETs, and its validity is examined. By examining the mathematical basis for the effective mass of electrons that have conduction band nonparabolicity, this paper also illuminates a model for the effective mass of holes having valence band nonparabolicity. 


\section{Modeling Nonparabolic Band Structure}

When an isotropic band structure is assumed, it is conventionally known that its form can be expressed generally as [10]

$$
E_{n-\mathrm{par}}\left(1+\alpha E_{n-\mathrm{par}}\right)=E_{\mathrm{par}}=\frac{\hbar^{2} k^{2}}{2 m^{*}}
$$

where $m^{*}$ is the effective mass of electrons in the isotropic band, $\alpha$ is the nonparabolic band factor, $E_{n \text {-par }}$ is the energy of electrons in the nonparabolic band, and $E_{\mathrm{par}}$ is the electron energy expression in the form of the parabolic band scheme. Here we reconsider how the realistic effective mass for device analysis should be estimated; the above simplified nonparabolic band model is assumed for the conduction band electrons of a three-dimensional quasi-free electron system.

When an external field effect is taken account of, we must examine whether the following formulation is theoretically valid or not:

$$
E_{n-\mathrm{par}}\left(1+\alpha E_{n-\mathrm{par}}\right)=E_{\mathrm{par}}+\beta E_{\text {ext }}
$$

where $E_{\text {ext }}$ is the perturbation energy corresponding to the 1st-order perturbation generated by the external electric field and $\beta$ is the perturbation factor. When (2) is valid, (2) can be rewritten as

$$
E_{n-\mathrm{par}}=\frac{\sqrt{1+4 \alpha\left(E_{\mathrm{par}}+\beta E_{\text {ext }}\right)}-1}{2 \alpha} .
$$

In order to examine the availability of (3), the right-hand side of (3) is changed into Tayler's power series.

$$
\begin{aligned}
& E_{n \text {-par }}=\frac{1}{2 \alpha}\left\{\left(1+\sum_{n=1}^{\infty} c_{n}\left(E_{\mathrm{par}}+\beta E_{\mathrm{ext}}\right)^{n}\right)-1\right\} \\
& =\frac{1}{2 \alpha}\left\{\left(1+2 \alpha\left(E_{\mathrm{par}}+\beta E_{\mathrm{ext}}\right)\right.\right. \\
& \left.\left.+\sum_{n=2}^{\infty} c_{n}\left(E_{\mathrm{par}}+\beta E_{\mathrm{ext}}\right)^{n}\right)-1\right\},
\end{aligned}
$$

where $c_{n}$ is the expansion coefficient and $c_{1}=2 \alpha$. If (4) is meaningful, $E_{n \text {-par }}$ should be the eigenvalue of the corresponding Schrödinger's equation. That is, (4) should be equivalent to the following operator representation:

$$
\begin{aligned}
& \widehat{H}_{n-\mathrm{par}}=\frac{1}{2 \alpha}\left\{\left(1+2 \alpha\left(\widehat{H}_{\mathrm{par}}+\beta \widehat{H}_{\mathrm{ext}}\right)\right.\right. \\
& \left.\left.\quad+\sum_{n=2}^{\infty} c_{n}\left(\widehat{H}_{\mathrm{par}}+\beta \widehat{H}_{\mathrm{ext}}\right)^{n}\right)-1\right\}=\widehat{H}_{\mathrm{par}}+\widehat{H}^{\prime},
\end{aligned}
$$

where all suffice and correspond to those in (4) and $\widehat{H}^{\prime}$ is the perturbation term that is expressed as

$$
\widehat{H}^{\prime}=\beta \widehat{H}_{\mathrm{ext}}+\frac{1}{2 \alpha} \sum_{n=2}^{\infty} c_{n}\left(\widehat{H}_{\mathrm{par}}+\beta \widehat{H}_{\mathrm{ext}}\right)^{n} .
$$

When we can assume that the amplitude of $\widehat{H}^{\prime}$ is smaller than that of $\widehat{H}_{\text {par }}$ [11], we approximately have

$$
\begin{aligned}
& E_{n \text {-par }}(k) \approx E_{\mathrm{par}}(k)-E_{C}+\beta E_{\mathrm{ext}}+\frac{1}{2 \alpha} \sum_{n=2}^{\infty} c_{n}\left(E_{\mathrm{par}}(k)\right. \\
& \left.-E_{C}+\beta E_{\mathrm{ext}}\right)^{n}=\frac{1}{2 \alpha}\{(1 \\
& +2 \alpha\left(\left(E_{\mathrm{par}}(k)-E_{C}+\beta E_{\mathrm{ext}}\right)\right) \\
& \left.\left.+\sum_{n=2}^{\infty} c_{n}\left(E_{\mathrm{par}}(k)-E_{C}+\beta E_{\mathrm{ext}}\right)^{n}\right)-1\right\} \\
& =\frac{\sqrt{1+4 \alpha\left(\left(E_{\mathrm{par}}(k)-E_{C}+\beta E_{\mathrm{ext}}\right)\right)}-1}{2 \alpha} .
\end{aligned}
$$

Equation (7) has the same form as (3). However, as the above formulation indicates, the meaning of (7) differs from that of (3) because (7) has been examined with the Hamiltonian operator representation in spite of the approximations used. Provided that prominent nonlinear effects are not significant, it can be concluded that (7) holds important physical meaning in terms of evaluating nonparabolic band effects on transport characteristics.

For the two-dimensional electron system, (7) can be rewritten as [11]

$$
\begin{aligned}
& E_{n-\mathrm{par}, n}(k) \\
& =\frac{\sqrt{1+4 \alpha\left(\left(E_{n}\left(k_{n}\right)-E_{C}+\beta E_{\text {ext }}\right)+E_{\text {free }}\left(k_{\|}\right)\right)}-1}{2 \alpha},
\end{aligned}
$$

for the $n$th subband attributed to the specific conduction band, where $E_{n}$ is the subband energy level of electrons, $k_{n}$ is the discrete wavenumber of the subband labeled " $n$ " in the quantum well, and $E_{\text {free }}\left(k_{\|}\right)$is the energy component of the transport direction. Equation (8) is valid only for $E_{1}-$ $E_{C} \gg E_{\text {ext }}$ based on the same assumption used for (7). Actually, it is easily found that $E_{\text {ext }}$ is much smaller than $E_{1}-E_{C}$ for electric fields less than $10^{7} \mathrm{~V} / \mathrm{cm}$ and $t_{S}<10 \mathrm{~nm}$ [10]; therefore, (8) is applicable to the analysis of nanoscale MOSFET characteristics.

The result described above yields an expression for the effective mass tensor $\left(m^{*}{ }_{n \text {-par, } i j}\right)$ for two-dimensional electron systems around the subband bottom as in [14]. Before using (8) for direct calculations, we review the conventional idea of the effective mass. We must recall the fact that electronic states with finite dimensions are inherently discrete; the difference from a very small size material is just the magnitude of $\Delta k_{i}$ between adjacent states. Naive calculation discards the 
term of the group velocity, but this is erroneous in terms of physics. The correct calculation result is given as

$$
\begin{aligned}
& \frac{1}{m_{n-\mathrm{par}_{i j}}}=\frac{1}{\hbar^{2}} \frac{\partial^{2} E_{n-\mathrm{par}, n}(k)}{\partial k_{j} \partial k_{i}}=(1 \\
& \left.+4 \alpha\left(\left(E_{j}\left(k_{j}\right)-E_{C}+\beta E_{\text {ext }}\right)+E_{\text {free }}\left(k_{\|}\right)\right)\right)^{-1 / 2} \\
& \quad \frac{1}{m_{\mathrm{par}, i j}}-2 \alpha(1 \\
& \left.+4 \alpha\left(\left(E_{j}\left(k_{j}\right)-E_{\mathrm{C}}+\beta E_{\mathrm{ext}}\right)+E_{\mathrm{free}}\left(k_{\|}\right)\right)\right)^{-3 / 2} \\
& \cdot v_{\mathrm{par}, j}^{c} v_{\mathrm{par}, i}^{c},
\end{aligned}
$$

where " $j$ " means confinement direction and $v_{\text {par, } j}^{c}$ is the "effective group velocity" along the direction labeled by " $j$. ." Given that label " $i$ " indicates the transport direction, we have

$$
v_{\mathrm{par}, i}^{c}=\frac{1}{\hbar} \frac{\partial E_{\mathrm{free}}\left(k_{\|}\right)}{\partial k_{i}} .
$$

Given that label " $j$ " represents the confinement direction, we have

$$
v_{\mathrm{par}, j}^{c}=\frac{1}{\hbar} \frac{\partial\left(E_{j}\left(k_{j}\right)+\beta E_{\mathrm{ext}}\right)}{\partial k_{j}}=\frac{\hbar k_{j}}{m_{j j}^{*}}+\frac{1}{\hbar} \frac{\partial \beta E_{\mathrm{ext}}}{\partial k_{j}},
$$

where $k_{j}$ is the discrete wavenumber and $m_{j j}^{*}$ is the effective mass along the confinement direction. The 2 nd term of the right-hand side is the perturbation term raised by the external field. When the semiconductor layer thickness is of the order of nanometers, the contribution of the 2nd term of (11) is quite small. Thus, the effective mass tensor $\left(m^{*}{ }_{n \text {-par }, i j}\right)$ value of low-dimensional electron systems can be calculated around the subband bottom [15]; the important point is the fact that the effective mass of electrons is larger by the factor of $\left(1+4 \alpha\left(E_{j}(0)-E_{C}+\beta E_{\text {ext }}\right)\right)^{1 / 2}$ than that estimated assuming a parabolic band.

\section{Calculation Results of Effective Mass of Low-Dimensionality Electrons}

Assuming a thin Si layer, we calculated effective mass values $\left(m_{l} / m_{0}\right.$ and $\left.m_{t} / m_{0}\right)$ for the (001) surface, where it is assumed that 2 -fold and 4 -fold $X$-band electrons are confined along the $\langle 001\rangle$ axis. Physical parameters assumed in calculations are summarized in Table 1. Figure 1 shows the effective mass of electrons occupying the ground state as a function of Si layer thickness $\left(t_{S}\right)$, where the nonparabolicity factor $\alpha$ is assumed to be $0.5 \mathrm{eV}^{-1}$ and, for comparison, 1st principle calculation results $[8,9]$ are also shown; the impact of band nonparabolicity on the effective mass of conduction band electrons appears when $t_{S}<5 \mathrm{~nm}[8,9]$. The present model successfully reproduces the 1st principle calculation results. Figure 2(a) shows the effective mass of electrons occupying the 1st excited state as a function of Si layer thickness. For comparison,
TABLE 1: Physical parameters assumed in calculations [12, 13].

\begin{tabular}{lcc}
\hline Notations & Values & Comments \\
\hline$m_{l}^{*} / m_{0}$ & 0.92 & $\begin{array}{c}\text { Longitudinal mass } \\
\text { of electrons } \\
\text { Transverse mass of } \\
\text { electrons }\end{array}$ \\
$m_{t}^{*} / m_{0}$ & 0.19 & $\begin{array}{c}\text { Heavy holes } \\
\text { Light holes }\end{array}$ \\
$m_{h h}^{*} / m_{0}$ & 0.49 & Free electrons \\
$m_{l h}^{*} / m_{0}$ & 0.16 & Bulk Si \\
$m_{0}$ & $9.1 \times 10^{-31}[\mathrm{~kg}]$ & \\
$n_{i}(@ 300 \mathrm{~K})$ & $9.7 \times 10^{9}\left[\mathrm{~cm}^{-3}\right]$ & \\
$E_{g}(@ 300 \mathrm{~K})$ & $1.1[\mathrm{eV}]$ & \\
Permittivity of $\mathrm{Si}\left(\varepsilon_{S}\right)$ & $12 \varepsilon_{0}[\mathrm{~F} / \mathrm{cm}]$ & \\
Permittivity of $\mathrm{SiO} \mathrm{O}_{2}$ & $3.8 \varepsilon_{0}[\mathrm{~F} / \mathrm{cm}]$ & \\
$\left(\varepsilon_{\text {OX }}\right)$ & $8.9 \times 10^{-14}[\mathrm{~F} / \mathrm{cm}]$ & \\
Permittivity of & & \\
vacuum $\left(\varepsilon_{0}\right)$ &
\end{tabular}

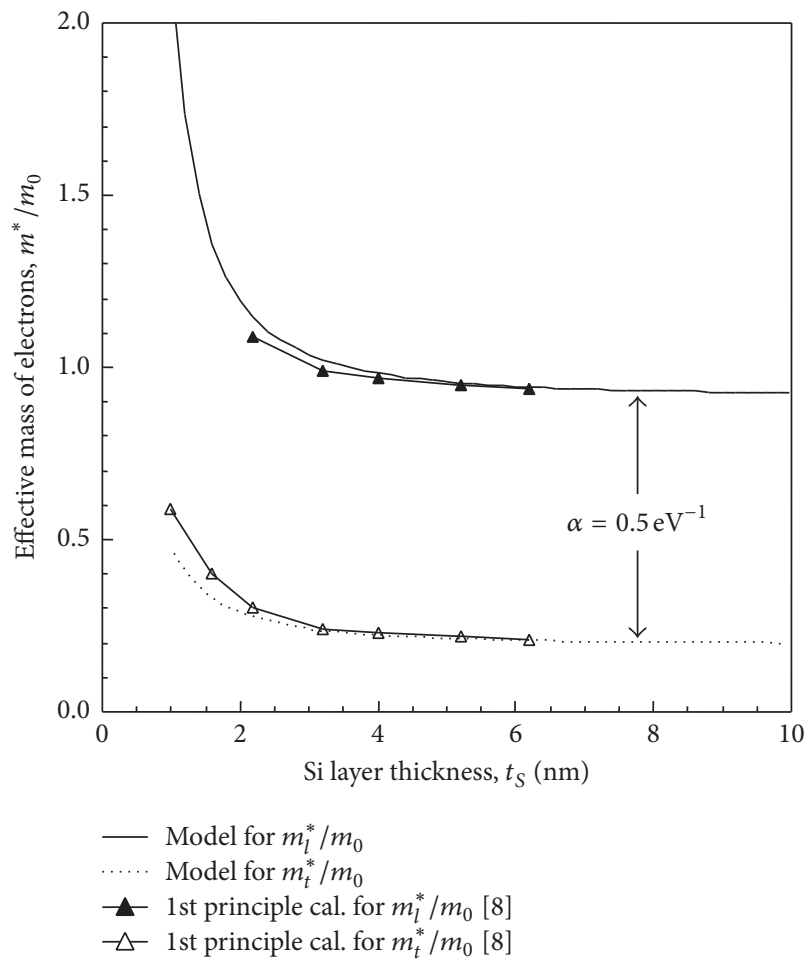

FIGURE 1: Calculated effective mass values of electrons occupying the ground state as a function of semiconductor layer thickness [11]. It is assumed that $\alpha=0.5 \mathrm{eV}^{-1}$ in order to calculate the nonparabolic band effect.

1st principle calculation results $[8,9]$ are also shown in Figure 2(a). The present model does not reproduce the 1st principle calculation results, so the conventional value of $\alpha$ is not appropriate. We, therefore, varied the value of $\alpha$ until the calculated curves fitted the 1st principle calculation results. Figure 2(b) shows the calculation results of the effective mass value of conduction band electrons occupying the 1st excited state, including the nonparabolicity effect, where it is assumed that $\alpha=0.1 \mathrm{eV}^{-1}$ for $m_{l} / m_{0}$ and $\alpha=0.05 \mathrm{eV}^{-1}$ for $m_{t} / m_{0}$. It 


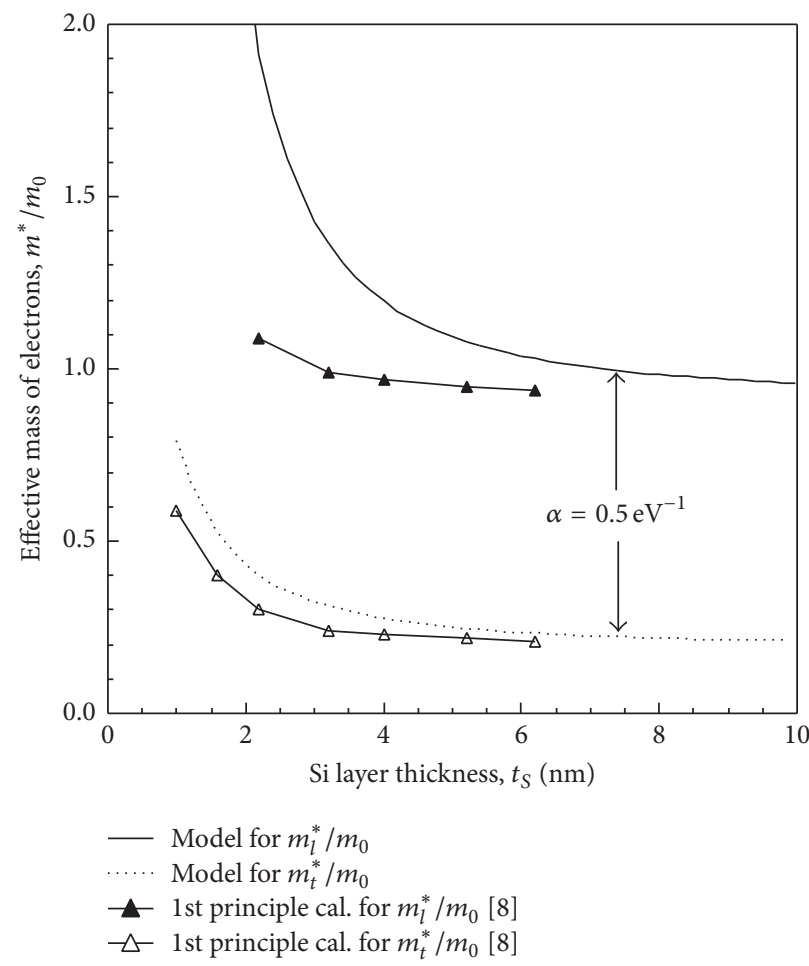

(a)

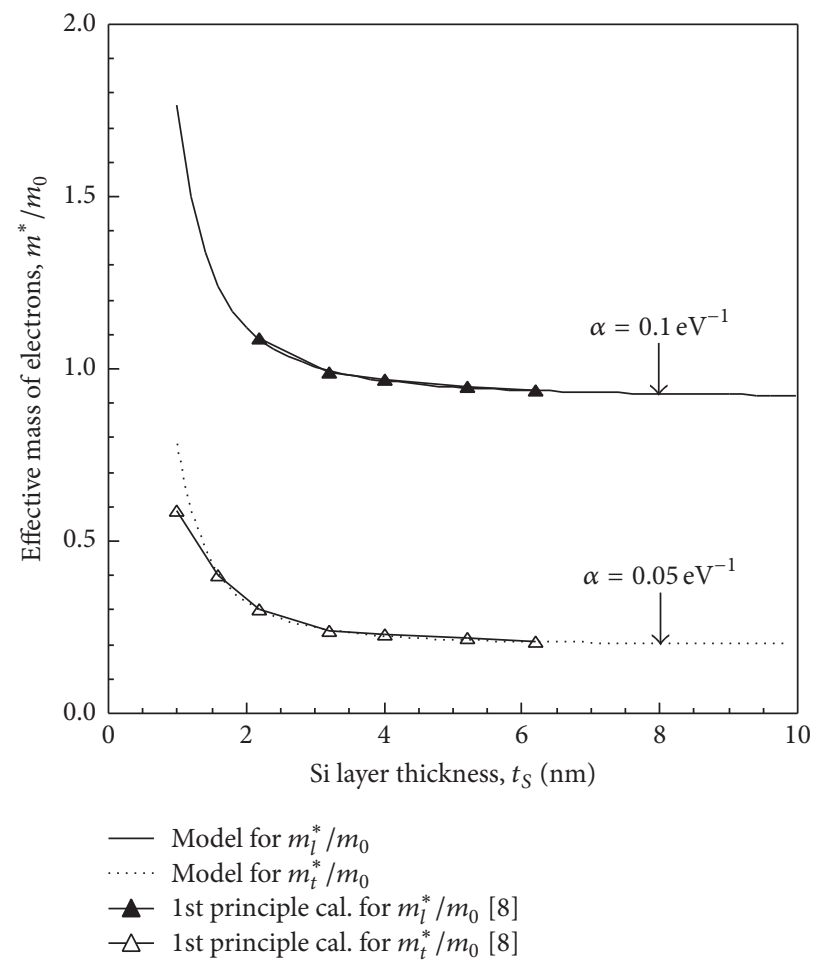

(b)

FIGURE 2: Calculated effective mass values of electrons occupying the 1st excited state as a function of semiconductor layer thickness [11]. (a) Effective mass dependence on Si layer thickness. It is assumed that $\alpha=0.5 \mathrm{eV}^{-1}$ in order to calculate the nonparabolic band effect. (b) Effective mass dependence on Si layer thickness. It is assumed that $\alpha=0.1 \mathrm{eV}^{-1}$ for $m_{l} / m_{0}$ and $\alpha=0.05 \mathrm{eV}^{-1}$ for $m_{t} / m_{0}$ in calculating the nonparabolic band effect.

is seen that the present model for effective mass successfully reproduces the theoretical simulation results. It is strongly suggested that the reason why the value of $\alpha$ for the 1st excited state is smaller than expected stems from the approximation that stripped higher-order expansion terms from (7).

\section{Applying Effective Mass Model to Express Threshold Voltage of Nanoscale MOSFETs}

Since the threshold voltage $\left(V_{\text {th }}\right)$ of ultrathin-body SOI MOSFETs is effectively ruled by the lowest energy level [3], it is easily anticipated that the impact of band nonparabolicity on $V_{\text {th }}$ is very significant. The author already clarified that the rise in threshold voltage stems from not only the quantummechanical mechanism [3] but also the semiclassical mechanism [5]. When it is assumed that the threshold voltage rising stems from the quantum confinement $[3,6,15], V_{\text {th }}$ for ultrathin-body $n$-channel SOI MOSFET can be expressed as [3]

$$
\begin{aligned}
& V_{\mathrm{th}}=V_{\mathrm{FB}}+\phi_{\mathrm{sth}}+\frac{Q_{B}}{C_{\mathrm{ox}}}, \\
& \phi_{\text {sth }}=\phi_{F, \mathrm{Q} M}+\left(\frac{E_{G}}{2 q}\right)+\frac{E_{n 1}-E_{C}}{q},
\end{aligned}
$$

where $V_{\mathrm{FB}}$ is the flat-band voltage, $C_{\mathrm{ox}}$ is the gate oxide capacitance per unit area, $Q_{B}$ is the depletion charge density, $\phi_{F, \mathrm{Q} M}$ is the "effective Fermi potential" including quantum effects (defined later), $E_{G}$ is the bandgap energy, and $E_{n 1}$ is the ground-state level energy of confined electrons in the conduction band. Fundamentally, the above expression for the threshold voltage of ultrathin-body SOI MOSFETs should yield the threshold voltage at low temperatures because the Fermi-Dirac function has a step-like function at low temperatures. At room temperature, the Fermi-Dirac function is thermally deformed, and the Fermi level does not simply define the threshold voltage; finite inversion layer charges are needed at the onset of the threshold [5]. From Boltzmann's approximation, at room temperature we have [16]

$$
\begin{aligned}
\phi_{F, Q M} & =\frac{E_{i}-E_{F}}{q} \\
& =\frac{1}{q}\left\{E_{i}-E_{p 1}+k_{B} T \ln \left(\frac{N_{A} t_{S}}{q D_{\mathrm{osp}} k_{B} T}\right)\right\},
\end{aligned}
$$

where $D_{\text {osp }}$ is the density of states of two-dimensional valence-band holes, $E_{p 1}$ is the ground-state level energy of confined holes in the valence band, and $N_{A}$ is the doping density; this expression is available for $E_{a}-E_{p 1} \gg k_{B} T$, where $E_{a}$ is the acceptor level energy. This expression is 
approximately derived from the following expression for intrinsic carrier density $\left(n_{i}^{*}\right)$, which should be called "effective intrinsic carrier density", in the quantum well defined at room temperature [16].

$$
n_{i}^{*}(T)=\left\{\frac{\left(D_{\text {osn }} D_{\text {osp }}\right)^{1 / 2} k_{B} T}{t_{S}}\right\} \exp \left[-\frac{E_{G}^{*}}{2 k_{B} T}\right],
$$

where $D_{\text {osn }}$ is the density of states of two-dimensional conduction band electrons; $E_{G}^{*}$ is the "effective bandgap energy" of the quantum well and can be expressed as

$$
E_{G}^{*}=E_{n 1}-E_{p 1}
$$

In calculating $V_{\text {th }}$ of a $p$-channel SOI MOSFET, we can also apply the present nonparabolic band model to holes (see the Appendix section).

On the other hand, $V_{\text {th }}$ for a quantum-wire SOI fin fieldeffect transistor (FinFET) is expressed as $[15,17]$

$$
\begin{aligned}
\phi_{\mathrm{sth}} & =\phi_{F, \mathrm{QM}}+\left(\frac{E_{G}}{2 q}\right)+\frac{E_{n, i j}-E_{C}}{q}, \\
E_{n, i j}-E_{C} & =\frac{h^{2}}{8}\left(\frac{i^{2}}{m^{*}{ }_{n z, i} t_{S}{ }^{2}}+\frac{j^{2}}{m^{*}{ }_{n x, j} w_{S}{ }^{2}}\right),
\end{aligned}
$$

where $h$ is Planck's constant, $t_{S}$ is fin height, $w_{S}$ is fin width, $E_{n, i j}$ is the quantum level energy, and $m^{*}{ }_{n x, j}\left(\right.$ or $\left.m^{*}{ }_{n z, i}\right)$ is the effective mass of electrons occupying the corresponding subband. In (16b), it is assumed that the Si wire is confined along $x$-axis and $z$-axis.

Calculated $V_{\text {th }}$ dependence on fin width of SOI FinFET two-dimensionally confined with (001) and (011) surfaces [6] and on SOI layer thickness of thin SOI MOSFET [7] is shown in Figure 3, where the top surface of the Si fin body is (001) and the side surface is (011). Physical parameters assumed in the calculations are summarized in Table 1. In this calculation of threshold voltage of FinFET, it is assumed that electrons of the Si body can be represented as quasi-two-dimensional system because the value of $t_{S}$ is larger than the minimal value of $w_{S}$. The conventional parabolic band model overestimates the quantum confinement effect in the range defined by sub-5-nm $t_{S}$. This suggests that the nonparabolicity should be taken into account when estimating the $V_{\text {th }}$ values of nanoscale SOI MOSFETs. On the other hand, experimental data of FinFET for $t_{S}>5 \mathrm{~nm}$ take values higher than two curves. It is anticipated, as one possibility, that fabricationinduced local variation of SOI layer thickness influences the threshold voltage of devices $[6,7,18]$.

Ge-on-insulator (GOI) devices are now attracting attention from the viewpoints of high-speed device applications. However, it is known that Ge demonstrates stronger conduction band nonparabolicity than Si. Therefore, the discussion given here is critical when considering nanoscale GOI device characteristics [19].

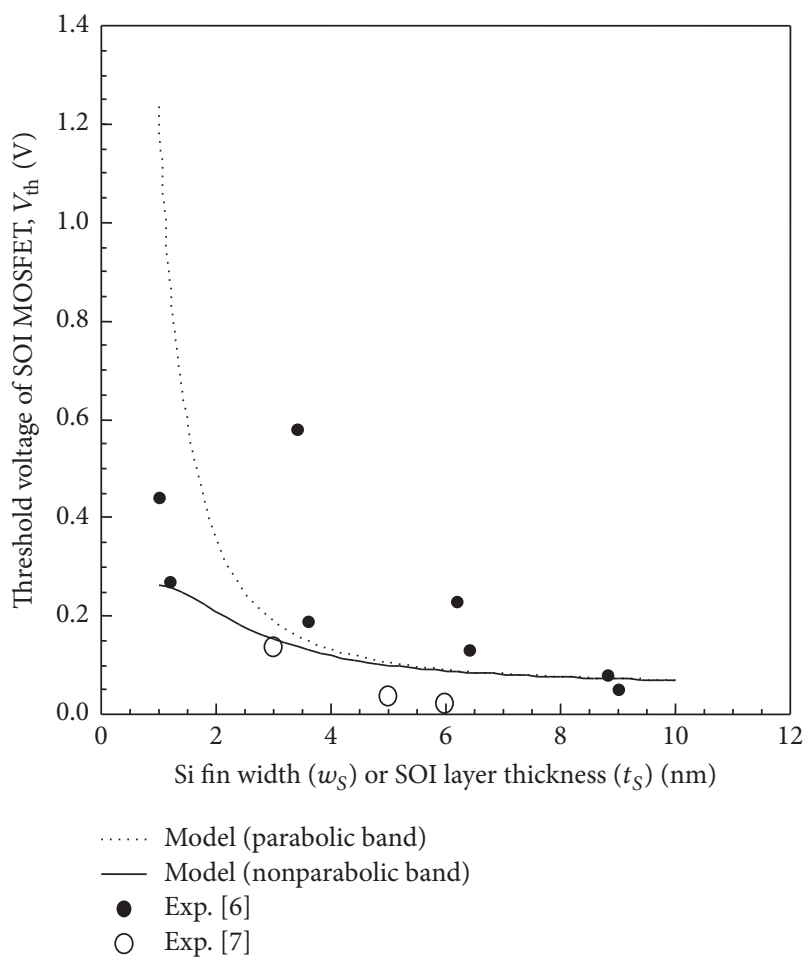

Figure 3: $V_{\text {th }}$ dependence on fin width or SOI layer thickness of Si SOI MOSFET device. The devices shown in [6] are twodimensionally confined with (001) and (011) surfaces. It is assumed that the gate oxide layer is $3 \mathrm{~nm}$ thick, the body silicon layer is $7 \mathrm{~nm}$ thick for [6], and $\alpha=0.5 \mathrm{eV}^{-1}$. The devices shown in [7] are twodimensionally confined with (001) surface. It is assumed that the gate oxide layer is $50 \mathrm{~nm}$ thick, the body silicon layer is ranging from $3 \mathrm{~nm}$ to $6 \mathrm{~nm}$ thick for [7], and $\alpha=0.5 \mathrm{eV}^{-1}$. It is also assumed that $m^{*} / m_{0}=0.916$ for (001) Si surface and $m^{*} / m_{0}=0.314$ for (011) Si surface.

\section{Conclusion}

This paper reconsidered the mathematical formulation of the conventional nonparabolic band model. Since the conventional simplified model for band nonparabolicity does not include the perturbation created by the external potential effect, we examined whether such perturbations could be added to the conventional model for convenience. When the perturbation energy is smaller than the unperturbed energy, the insertion of a perturbation term into the conventional expression for the nonparabolic band model is valid; it was confirmed that this approximation is acceptable given a sub-10-nm-thick Si layer surrounded by an $\mathrm{SiO}_{2}$ layer. The major discussion focused on the low-dimensional electron system confined by an insulator barrier. For the purpose of verifying this consideration, we addressed the influence of band nonparabolicity on the threshold voltage of Sibased nanoscale SOI FinFETs; calculation results yielded by the proposed model were compared to experimental results and the validity of the model proposed here was confirmed. 


\section{Appendix}

\section{Modeling Nonparabolicity of Valence Band Holes}

In the valence band, it is well known that the nonparabolic band energy dispersion relation of holes can be approximately expressed as [10]

$$
\begin{aligned}
& E_{\text {hole,nonpara }}(k)=\frac{\hbar^{2}}{2 m_{0}}\left(A k^{2}\right. \\
& \left.\quad \pm\left\{B^{2} k^{4}+C^{2}\left(k_{x}^{2} k_{y}^{2}+k_{y}^{2} k_{z}^{2}+k_{z}^{2} k_{x}^{2}\right)\right\}^{1 / 2}\right),
\end{aligned}
$$

where $A, B$, and $C$ are constants with values of $4.22,0.78$, and 4.80 , respectively [10]. When the system is confined along $z$-axis, $k_{z}$ is discrete and its notation should be replaced with $k_{z, n}(n=1,2,3 \ldots)$. Equation (A.1) must be replaced with the following:

$$
\begin{gathered}
E_{2 D \text {-hole,nonpara }}=\frac{\hbar^{2}}{2 m_{0}}\left(A\left(k_{x}^{2}+k_{y}^{2}+k_{z, n}^{2}\right)\right. \\
\pm\left\{B^{2}\left(k_{x}^{2}+k_{y}^{2}+k_{z, n}^{2}\right)^{2}\right. \\
\left.\left.+C^{2}\left(k_{x}^{2} k_{y}^{2}+k_{y}^{2} k_{z, n}^{2}+k_{z, n}^{2} k_{x}^{2}\right)\right\}^{1 / 2}\right) .
\end{gathered}
$$

Therefore, the effective mass of holes $\left(m_{h, z z}^{*}\right)$ in the valence band can be expressed as

$$
\begin{aligned}
& \frac{1}{m_{h, z z}^{*}\left(k_{x}, k_{y}, k_{z, n}\right)}=\frac{1}{\hbar^{2}} \frac{\partial^{2} E_{2 D \text {-hole,nonpara }}(k)}{\partial k_{z, n}^{2}}=\frac{1}{m_{0}}\left(A \mp \frac{\left\{2 B^{2}\left(k_{x}^{2}+k_{y}^{2}+k_{z, n}^{2}\right) k_{z, n}+C^{2}\left(k_{y}^{2}+k_{x}^{2}\right) k_{z, n}\right\}^{2}}{2\left\{B^{2}\left(k_{x}^{2}+k_{y}^{2}+k_{z, n}^{2}\right)^{2}+C^{2}\left(k_{x}^{2} k_{y}^{2}+k_{y}^{2} k_{z, n}^{2}+k_{z, n}^{2} k_{x}^{2}\right)\right\}^{3 / 2}}\right. \\
& \left. \pm \frac{2 B^{2}\left(k_{x}^{2}+k_{y}^{2}+3 k_{z, n}^{2}\right)+C^{2}\left(k_{y}^{2}+k_{x}^{2}\right)}{2\left\{B^{2}\left(k_{x}^{2}+k_{y}^{2}+k_{z, n}^{2}\right)^{2}+C^{2}\left(k_{x}^{2} k_{y}^{2}+k_{y}^{2} k_{z, n}^{2}+k_{z, n}^{2} k_{x}^{2}\right)\right\}^{1 / 2}}\right) .
\end{aligned}
$$

At the bottom of valence band, $k_{x}=k_{y}=0$, and we have

$$
\frac{1}{m_{h, z z}^{*}\left(0,0, k_{z, n}\right)}=\frac{1}{m_{h, z z}^{*}}=\frac{1}{m_{0} /(A \pm B)} \text {. }
$$

From (A.4), we have $m_{h, z z}^{*}=0.20 m_{0}$ for the light hole and $m_{h, z z}^{*}=0.29 m_{0}$ for the heavy hole. Thus, when we use those effective mass values, the ground-state level energy $E_{p, 1}$ is given by

$$
E_{V}-E_{p, 1}=\frac{\hbar^{2} \pi^{2}}{2 m_{h, z z}^{*} t_{S}^{2}} .
$$

Equation (A.4) is an approximate expression for the effective mass of holes without any $t_{S}$ dependence. However, this is not an appropriate result because $m^{*}{ }_{h, z z}$ values are not accurate for both light holes and heavy holes as shown above. Accordingly, we have to derive a new $t_{S}$-dependent expression for the effective mass of holes.

Our solution is to rewrite (A.1) as a function of parabolic band energy $E_{\text {holes,para }(k)}$ :

$$
\begin{gathered}
E_{\text {hole,nonpara }}(k)=\frac{m_{h, \text { para }}^{*} E_{\text {hole,para }}(k)}{m_{0}}\left(A \pm\left\{B^{2}\right.\right. \\
+\frac{C^{2}}{2}(1 \\
\left.\left.\left.-\frac{E_{x, \text { hole,para }}^{2}+E_{y, \text { hole,para }}^{2}+E_{z, \text { hole,para }}^{2}}{E_{\text {hole,para }}^{2}(k)}\right)\right\}^{1 / 2}\right),
\end{gathered}
$$

where we assume that

$$
\begin{aligned}
E_{\text {hole,para }}(k) & =\frac{\hbar^{2} k^{2}}{2 m_{h, \text { para }}^{*}} \\
& =E_{x, \text { hole,para }}+E_{y, \text { hole,para }}+E_{z, \text { hole,para }} .
\end{aligned}
$$

Since we have $\left(E_{x \text {,hole,para }}^{2}+E_{y \text {,hole,para }}^{2}+E_{z \text {,hole,para }}^{2}\right) /$ $E_{\text {hole,para }}^{2}(k) \approx 1 / 3$ in $(6),(6)$ can be approximately rewritten as

$$
\begin{gathered}
E_{\text {hole,nonpara }}(k) \cong \frac{m_{h, \text { para }}^{*} E_{\text {hole,para }}(k)}{m_{0}}(A \\
\pm \sqrt{B^{2}+\frac{C^{2}}{2} \mp \frac{1}{\sqrt{B^{2}+C^{2} / 2}}} \\
\left.\cdot \frac{E_{x, \text { hole,para }}^{2}+E_{y, \text { hole,para }}^{2}+E_{z \text {,hole,para }}^{2}}{2 E_{\text {hole,para }}^{2}(k)}\right) .
\end{gathered}
$$

Here, we consider the case of a two-dimensional hole system in which the confinement is along $z$-axis; from (A.7), we have

$$
\begin{aligned}
E_{z, 2 D \text {-hole,para }}\left(k_{z, n}\right)=\frac{\hbar^{2} k_{z, n}^{2}}{2 m_{h, \text { para }}^{*}} \equiv \frac{\hbar^{2} n^{2} \pi^{2}}{2 m_{h, \text { para }}^{*} t_{S}^{2}}, \\
\quad(n=1,2,3 \ldots),
\end{aligned}
$$

where a parabolic band hole system is assumed for simplicity; it is assumed that $m_{h \text {,para }}^{*}$ is the parabolic band mass of holes; 
that is, $m_{h \text {,para }}^{*}=\left(m_{l h}^{* 3 / 2}+m_{h h}^{* 3 / 2}\right)^{2 / 3}$. Combining (A.8) with (A.9) yields the following expression for the two-dimensional hole system.

$$
\begin{gathered}
E_{2 D \text {-hole, nonpara }}(k) \cong \frac{m_{h, \text { para }}^{*} E_{2 D \text {-hole,para }}(k)}{m_{0}}(A \\
\pm \sqrt{B^{2}+\frac{C^{2}}{2} \mp \frac{1}{\sqrt{B^{2}+C^{2} / 2}}} \\
\left.\cdot \frac{E_{x, \text { hole,para }}^{2}+E_{y, \text { hole,para }}^{2}+E_{z, 2 D \text {-hole,para }}^{2}}{2 E_{2 D \text {-hole,para }}^{2}(k)}\right) .
\end{gathered}
$$

In a practical two-dimensional system, carriers are usually confined by barriers and the external field $\left(F_{\text {ext }}\right)$. Therefore, we have to introduce a perturbation $\left(E_{\text {ext }}\right)$ to represent the effect of the external field into (A.10). Accordingly, we have

$$
\begin{aligned}
& E_{2 D \text {-hole,nonpara }}(k) \cong \frac{m_{h, \text { para }}^{*} E_{2 D \text {-hole,para }}(k)}{m_{0}}\left(K_{1} \mp K_{2}\right. \\
& \left.\cdot \frac{E_{x, \text { hole,para }}^{2}+E_{y, \text { hole,para }}^{2}+\left(E_{z, 2 D \text {-hole,para }}+E_{\text {ext }}\right)^{2}}{2 E_{2 D \text {-hole,para }}^{2}(k)}\right), \\
& E_{2 D \text {-hole,para }}(k)=E_{x, \text { hole,para }}+E_{y, \text { hole,para }}+E_{z, 2 D \text {-hole,para }}(\text { A.11 }) \\
& +E_{\text {ext }}, \\
& K_{1}=A \pm \sqrt{B^{2}+\frac{C^{2}}{2}} \\
& K_{2}=\frac{1}{\sqrt{B^{2}+C^{2} / 2}},
\end{aligned}
$$

where $E_{\text {ext }}$ stands for the external energy stemming from the external field $\left(F_{\text {ext }}\right)$. This expression is physically reasonable when $E_{\text {ext }} \ll E_{z, 2 D \text {-hole,para }}[20]$.

\section{Competing Interests}

The author declares that there are no competing interests.

\section{Acknowledgments}

A part of this study was partially conducted by MEXTSupported Program for the Strategic Research Foundation at Private Universities, "creation of $3 \mathrm{~d}$ nano-micro structures and its applications to biomimetics and medicine," 20152019.

\section{References}

[1] J. P. Colinge, Silicon-on-Insulator Technology: Materials to VLSI, Springer, New York, NY, USA, 3rd edition, 2004.

[2] J.-P. Colinge, Ed., FinFETs and Other Multi-Gate Transistors, Springer, New York, NY, USA, 2008.
[3] Y. Omura, S. Horiguchi, M. Tabe, and K. Kishi, "Quantummechanical effects on the threshold voltage of ultrathin-SOI nMOSFET's," IEEE Electron Device Letters, vol. 14, no. 12, pp. 569-571, 1993.

[4] Y. Ishikawa, T. Ishihara, T. Tsuchiya, and M. Tabe, "XPS and $\mathrm{I}-\mathrm{V}$ studies on quantum mechanical effects in ultrathin si layer of SOI structure," in Proceedings of the Abstract Silicon Nanoelectronics Workshop, pp. 14-15, Kyoto, Japan, June 2001.

[5] Y. Tamara and Y. Omura, "Empirical quantitative modeling of threshold voltage of sub-50-nm double-gate silicon-oninsulator metal-oxide-semiconductor field-effect transistor," Japanese Journal of Applied Physics, Part 1: Regular Papers and Short Notes and Review Papers, vol. 45, no. 4, pp. 3074-3078, 2006.

[6] H. Majima, H. Ishikuro, and T. Hiramoto, "Experimental evidence for quantum mechanical narrow channel effect in ultra-narrow MOSFET's," IEEE Electron Device Letters, vol. 21, no. 8, pp. 396-398, 2000.

[7] T. Ernst, S. Cristoloveanu, G. Ghibaudo et al., "Ultimately thin double-gate SOI MOSFETs," IEEE Transactions on Electron Devices, vol. 50, no. 3, pp. 830-838, 2003.

[8] K. Nehari, N. Cavassilas, J. L. Autran, M. Bescond, D. Munteanu, and M. Lannoo, "Influence of band structure on electron ballistic transport in silicon nanowire MOSFET's: an atomistic study," Solid-State Electronics, vol. 50, no. 4, pp. 716-721, 2006.

[9] P. V. Sushko and A. L. Shluger, "Electronic structure of insulatorconfined ultra-thin Si channels," Microelectronic Engineering, vol. 84, no. 9-10, pp. 2043-2046, 2007.

[10] B. K. Ridley, Quantum Processes in Semiconductors, Clarendon, Oxford, UK, 2nd edition, 1988.

[11] Y. Omura, "Extension of analytical model for conduction band non-parabolicity to transport analysis of nano-scale metaloxide-semiconductor field-effect transistor," Journal of Applied Physics, vol. 105, no. 1, p. 014310, 2009.

[12] S. M. Sze and K. K. Ng, Physics of Semiconductor Devices, Wiley InterScience, 3rd edition, 2007.

[13] C. Jacoboni and L. Reggiani, "The Monte Carlo method for the solution of charge transport in semiconductors with applications to covalent materials," Reviews of Modern Physics, vol. 55, no. 3, pp. 645-705, 1983.

[14] S. Jin, M. V. Fischetti, and T.-W. Tang, "Modeling of electron mobility in gated silicon nanowires at room temperature: surface roughness scattering, dielectric screening, and band nonparabolicity," Journal of Applied Physics, vol. 102, no. 8, Article ID 083715, 2007.

[15] Y. Omura, T. Ishiyama, M. Shoji, and K. Izumi, "Quantum Mechanical Transport Characteristics in Ultimately Miniaturized MOSFETs/SIMOX," in Proceedings of the 10th International Symposium on SOI Technology and Development, vol. PV96-3, pp. 199-205, Electrochemical Society, March 1996.

[16] Y. Omura, Soi Lubistors, chapter 10, IEEE/Wiley, 1st edition, 2013.

[17] R. Granzner, F. Schwierz, and V. M. Polyakov, "An analytical model for the threshold voltage shift caused by twodimensional quantum confinement in undoped multiple-gate MOSFETs," IEEE Transactions on Electron Devices, vol. 54, no. 9, pp. 2562-2565, 2007.

[18] Y. Omura and M. Nagase, "Low-temperature drain current characteristics in sub-10-nm-thick SOI nMOSFET's on SIMOX (separation by IMplanted OXygen) substrates," Japanese Journal of Applied Physics, Part 1: Regular Papers \& Short Notes \& Review Papers, vol. 34, no. 2B, pp. 812-816, 1995. 
[19] L. Pantisano, L. Trojman, J. Mitard et al., "Fundamentals and extraction of velocity saturation in Sub-100 nm (110)-Si and (100)-G," in Proceedings of the Abstract International Symposium on VLSI Technology, Systems, and Applications, pp. 52-53, 2008.

[20] Y. Omura, "Modeling hole effective mass of Si modulated by external field," in Proceedings of the 15th Silicon Nanoelectronics Workshop (SNW '10), pp. 65-66, Honolulu, Hawaii, USA, June 2010. 


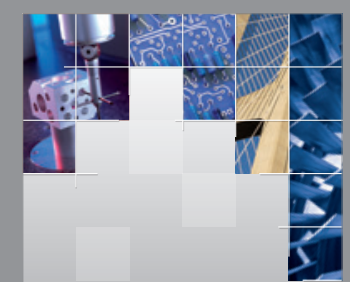

\section{Enfincering}
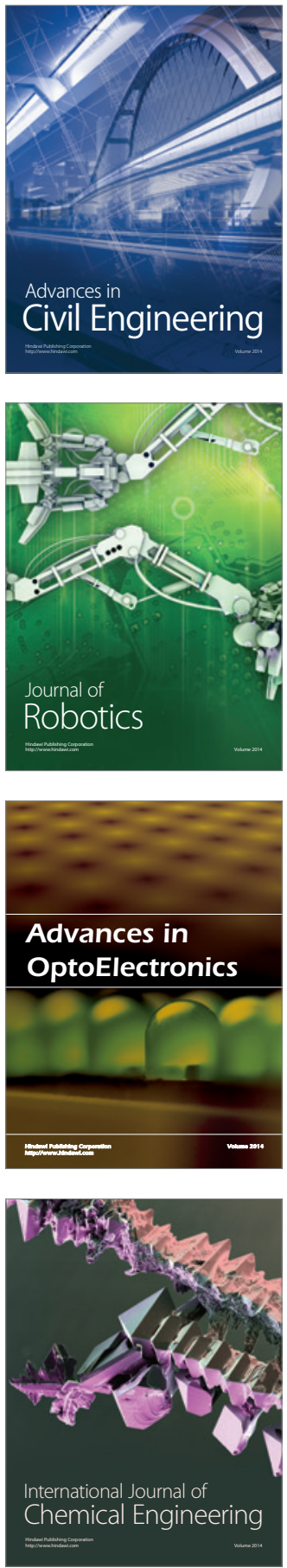

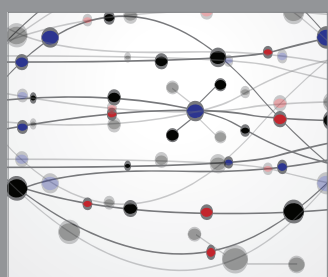

The Scientific World Journal

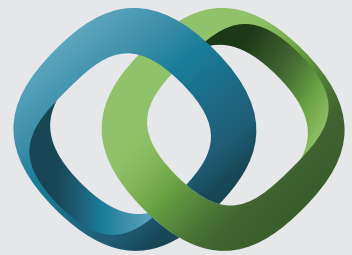

\section{Hindawi}

Submit your manuscripts at

http://www.hindawi.com
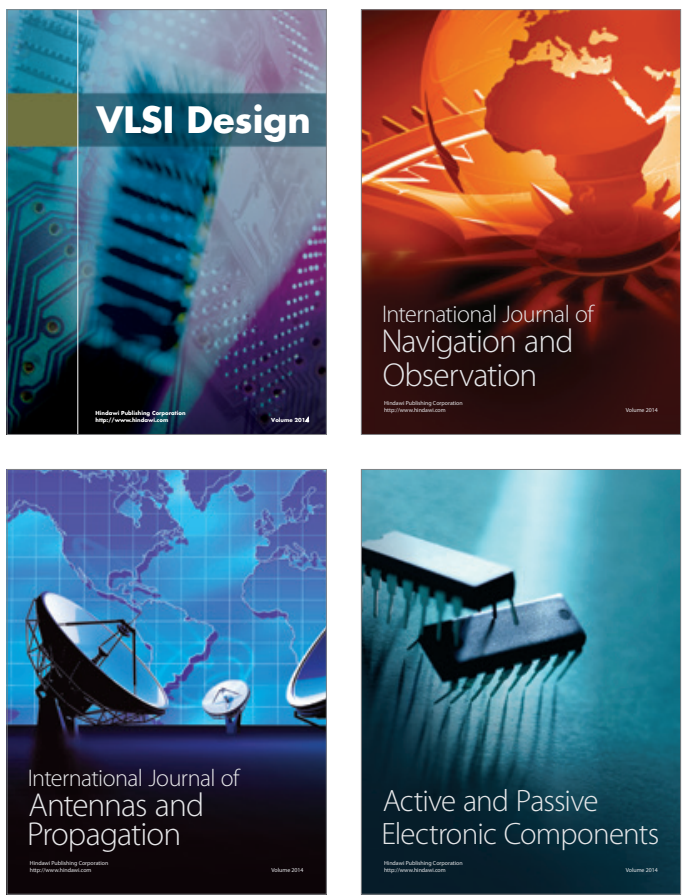
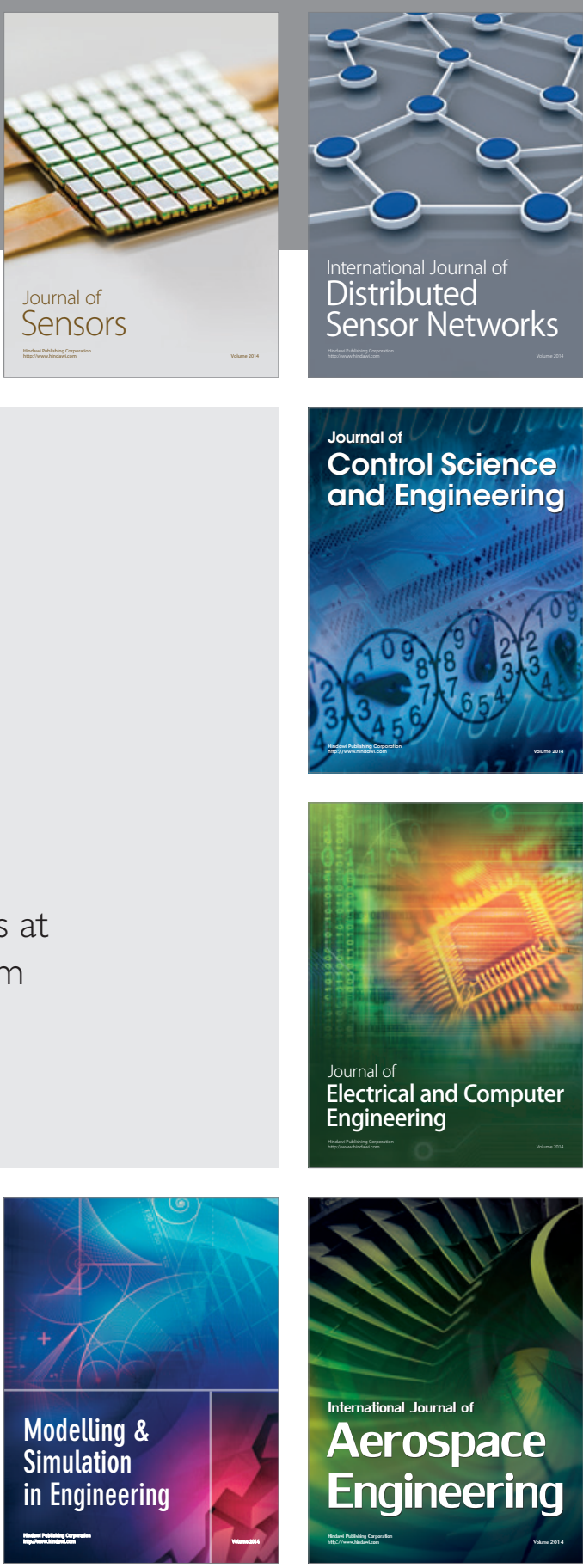

International Journal of

Distributed

Sensor Networks

Journal of

Control Science

and Engineering
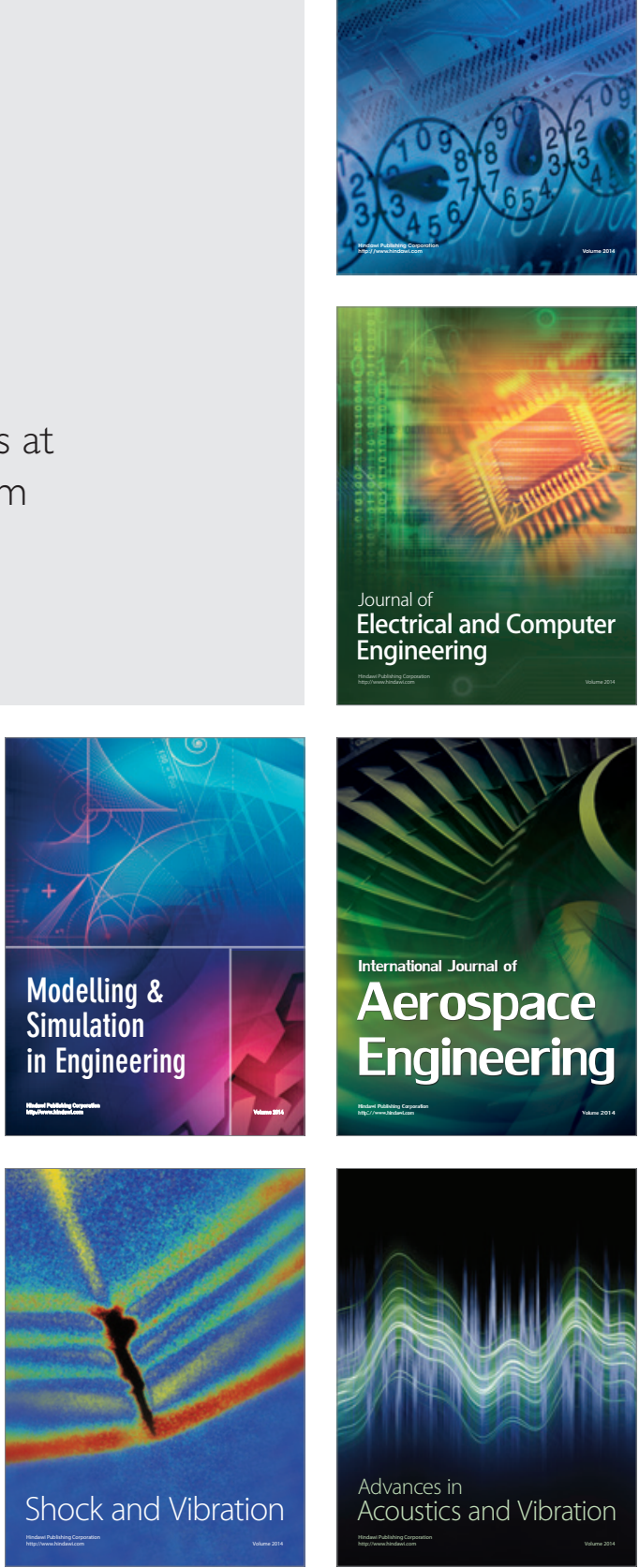